\title{
Microstructure evolution in chelated partially hydrolysed alumina sols during sonogelling
}

\author{
MANOJ M HARIDAS and JAYESH R BELLARE* \\ Department of Chemical Engineering, Indian Institute of Technology, Powai, Bombay \\ 400076, India \\ MS received 10 January 1996; revised 3 October 1996
}

\begin{abstract}
Optically transparent alumina sonogels with optical transmittance of over $90 \%$ have been prepared by chemically modifying a partially hydrolysed aluminium sec-butoxide precursor with ethyl acetoacetate. Chelating the alkoxide in a 1:2 molar ratio has permitted an enhanced control over microstructural changes during the sol to sonogel synthesis. Particle size and morphology changes during controlled sonogelling indicate that the sol to sonogel evolution in chelated alkoxides occurs by a dissolution-nucleation process in which the chelated species dissolves and aluminium hydroxide nucleates. The chelated agglomerates break down with increasing amount of water, and finally dissolve into the alcohol rich medium. The breakdown of chelated agglomerates is accompanied by the formation of nanosized aluminium hydroxide particles which grow with water addition into aggregated micron sized, spherical species. The chelated agglomerates evolve from a spherical geometry to a cylindrical morphology to a tapelike structure with a preferred orientation, and finally to a wavy sheet-like matrix. The nano-aluminium hydroxide particles increase in size at every water addition and grow to about $1 \mu \mathrm{m}$ at the sonogel point.
\end{abstract}

Keywords. Sol-gel: microstructure; alkoxide; chelate; cryo-SEM; agglomerate; nanoalumina; evolution; viscosity: QELS.

\section{Introduction}

Aluminium alkoxides are aluminium salts of alcohols known for their high affinity for water (Mehrotra and Mehrotra 1961; Sakka and Yoko 1991). Uncontrolled hydrolysis of these precursors results in a gelatinous precipitate of aluminium hydroxide having an opaque (Yoldas 1973), porous (Yoldas 1975), irregular microstructure (Ogihara et al 1991) and a broad particle size distribution (Ogihara et al 1991). Since the processing variables directly affect the microstructure of the ceramic powder, a microstructural control over the sol to gel transition essentially involves a control over the rate of hydrolysis of the alkoxide. Hydrolysis control can be done either by a slow water addition under controlled environmental, temperature and humidity conditions (Yoldas 1973; Leaustic and Riman 1991) or, by an extremely efficient sonication which prevents a local water attack (Yoldas 1975). However, this is best achieved by using chelating agents like ethyl acetoacetate (EAA) which shield the extremely electronegative $\mathrm{O}-\mathrm{Bu}^{s}$ linkage, modify the alkoxide precursor and make the precursor inert to a nucleophilic attack (Wengrovius et al 1986; Sanchez et al 1988; Schmidt 1988; Nass and Schmidt 1990; Yogo and Iwahara 1992; Heinrich et al 1994). Some chelating agents can be removed at a later stage in the processing without leaving any trace of impurities behind. Beta-diketones belong to such a family of chelating agents and this area has been of research focus for some time now. Microstructural

* To whom all correspondences should be addressed. 
evolution during alkoxide sonogelling has not been investigated earlier. Proposing a sonogelling mechanism has formed the thrust of our experimental investigation.

The present investigation concentrates on the mechanisms of alkoxide sonogelling using a chelate ratio of 1:1 to 1:3. The focus of this research is to evaluate a structureproperty-processing relation in the $5 \mathrm{~nm}-10 \mu \mathrm{m}$ size range from viscosity, optical transmittance, QELS and cryo-SEM analyses. The QELS observations for particle size changes in the 5-1000 nm size range and cryo-SEM observations for particle size, distributions and morphology changes in the $400 \mathrm{~nm}-10 \mu \mathrm{m}$ region were used to corroborate findings in their mutually overlapping regions and present an evolutionary model at the microscopic to macroscopic scale.

\section{Experimental}

\subsection{Materials}

The aluminium sec-butoxide (ASB) used in the experiments was of $97 \%$ assay (Fluka). The isopropyl alcohol (IPA) (Fluka) with a $96 \%$ assay was dried by a 4 -hour storage in a desiccator containing calcium oxide previously heated to $800^{\circ} \mathrm{C}$. The ethyl acetoacetate (EAA) (Spectrochem) with a $96 \%$ assay was used as received. Milli-Q water $(18 \mathrm{M} \Omega-\mathrm{cm})$ was used to hydrolyse the chelated alumina sols.

\subsection{Methods}

2.2a Preparation: About $3.0 \mathrm{~g}$ of ASB were weighed out in a sample bottle in a glove box. Details on the hydrolysis control measures are elaborated elsewhere (Haridas and Bellare 1995a, b, c). About $15 \mathrm{ml}$ of previously dried IPA were added to it and the mix ultrasonically agitated for $600 \mathrm{sec}$ on a Branson- 450 probe type sonicator. To counteract the effects of heating, and to eliminate the generation of the presently undesirable high temperature phases of aluminium hydroxide (Yoldas 1973), the alcoholic alkoxide was enclosed in a cooling jacket to maintain a temperature of $28^{\circ} \mathrm{C}$ throughout the sonication period.

A controlled hydrolysis on the unchelated reactants was performed by intimately sonicating the ASB and the IPA in a $35 \mathrm{ml}$ sample bottle. The sample bottle was placed in a glove box which was flushed with argon gas. The temperature of the reactants in the sample bottle was kept constant at $28^{\circ} \mathrm{C}$ using a cooling jacket. Air at an R.H. of $60 \%$ was passed into the glove box through a $0.5 \mathrm{~cm}$ nozzle for $60 \mathrm{sec}$ so as to initiate an alkoxide hydrolysis under controlled conditions of moisture, temperature and ultrasonication without external water addition. Visually, there appeared to be no microstructural changes, as gauged by no-noticeable drop in transparency in the liquid sample. As reported in literature (Mehrotra and Mehrotra 1961; Yoldas 1973, 1975; Debsikdar 1985; Strawbridge and James 1986; Segal 1989; Ogihara et al 1991; Ching and Klein 1988a, b; Jones 1989; Ichinose et al 1992), the factors which ensure a control over the alkoxide hydrolysis are: (i) the amount of alkoxide, (ii) the amount of moisture available to the alkoxide, (iii) the duration of moisture availability, (iv) the temperature of the reactants, ( $v$ ) the amount of alcohol present and (vi) the amount of hydrolysis retarders, if present. Since these factors are known as a result of experimental design, it can be concluded that hydrolysis is controlled. In our experiments, all batch weighings 
were performed in the inert atmosphere of the glove box in presence of $\mathrm{P}_{2} \mathrm{O}_{5}$ desiccant.

For a stoichiometric 1:1 chelate ratio, about $1.5 \mathrm{ml}$ of EAA were added to the alkoxide using a micropipette. Continuous sonication for about $1200 \mathrm{sec}$ was performed to complete the alkoxide chelation (Wengrovius et al 1986; Schmidt 1988; Sanchez et al 1988; Babonneau et al 1990). For a 1:2 chelate ratio, about $3.0 \mathrm{ml}$ of ethyl acetoacetate were added under similar sonication and temperature conditions.

$2.2 \mathrm{~b}$ Reaction: From prior experiments, it was ascertained that $1: 1$ chelated sols, sonogel on the addition of $1.0 \mathrm{ml}$ water when it was added at the rate of $0.2 \mathrm{ml}$ every $600 \mathrm{sec}$ (or $0.4 \mathrm{ml}$ per hour per gram of ASB) under continuous sonication conditions. Similarly, 1:2 chelated sols, sonogel at $1.8 \mathrm{ml}$ of water addition. After about 50 trial transmittance studies (Haridas and Bellare 1995a,b,c), it was determined that $15 \mathrm{ml}$ IPA per $3.0 \mathrm{~g}$ batch of ASB gave a sonogel transmittance of over $90 \%$ and this was taken as the basis for all alcohol additions.

Water addition was stopped at the sonogel point which occurred at $1.0 \mathrm{ml}$ for a $1: 1$ chelate and $1.8 \mathrm{ml}$ for a 1:2 chelate as indicated in previously conducted tests. Batches of EAA chelated ASB with $15 \mathrm{ml}$ IPA, at $0.2 \mathrm{ml}$ water increments, were then taken up for microstructural characterization during the sol to sonogel transition.

The samples were allowed to stand in a glove box having $\mathrm{P}_{2} \mathrm{O}_{5}$ desiccant under controlled humidity conditions and left undisturbed until the partially hydrolysed sols gelled.

2.2 c Characterization: To characterize the entire sol-to-sonogel synthesis at differing chelate ratios, four different techniques were used on the basis of their direct or indirect ability to reveal the microstructure. Viscosity (Brookfield viscometer: 74R using an LV-18 spindle) studies were performed on all samples during water addition, at an rpm ranging from 0.3 to 60 which corresponds to a shear rate of $120-1500 \mathrm{sec}^{-1}$. Optical transmittance investigations on graded sol samples were performed on a Shimadzu-160 spectrophotometer using matched pair quartz cuvettes, at a $10.0 \mathrm{~mm}$ path length at a typical source wavelength of $520 \mathrm{~nm}$. Quasielastic light scattering studies (Brookhaven Instruments Corp. BI-90) were carried out on all sol samples, and were essential to learn about particle size changes during sonogelling. For a morphological investigation into the gelling process and to propose a 'directly' observed gelling model, cryo-SEM studies were carried out on an electron microscope equipped with a cold stage.

$2.2 \mathrm{~d}$ Electron microscopy characterization: Cryo-SEM was performed on a JEOL:JSM 6400 microscope using fast-freeze, freeze-fracture and direct visualization techniques for imaging, as it has been recently reported (Bhadriraju and Bellare 1993; Haridas et al 1995). Microstructural detail i.e. contrast, comes from an intensity difference of the secondary electrons generated due to topography and edges. The sites with a higher concentration of secondary electrons appear bright in the photomicrograph and generate a good topological contrast over the dark matrix. Some contrast enhancement, to get a well defined particulate morphology is done by subtracting the adjoining grey shades from the images, so as to achieve an improvement in image quality. The details of the cryo-SEM technique have been well established, and the methodology as applied to fluid, labile systems is reported elsewhere (Haridas et al 1995). 
The sols in various stages of sonogelling were collected, and $100 \mu \mathrm{l}$ from each sample extracted using a microsyringe. The sample was sandwiched between two 300 mesh, nickel, TEM grids supported on copper plates. The sandwich was plunged into melting freon at $-160^{\circ} \mathrm{C}$, at high speeds to initiate a rapid solidification to a glassy state. Crystallization was prevented, and the liquid state of matter preserved within the rapidly solidified sample. The samples were transferred to the airlock chamber of the electron microscope. Here, they were fractured with a specially designed fracture knife, so as to expose the sol microstructure to the incident electron beam. The samples were coated with about $0.1 \mu \mathrm{m}$ of gold, by evaporation and viewed for magnifications between $500 \times$ and $10,000 \times$. This technique has recently been established as one which makes direct, artifact-free imaging of colloidal microstructures feasible and details are reported elsewhere (Bellare 1988).

Microstructures seen in alkoxides spanned a very large range in terms of size (Haridas et al 1995). The cryo-SEM technique was more suitable for the larger: micron and sub-micron sized particles, due to effects arising from a combination of microscopic resolution and sample fixation techniques. On the other hand, QELS was more suitable for the smaller, nanometer sized particles seen during the early stages of hydrolysis, and less useful for the micron sized particles (Finsy 1994). The chelated agglomerate size was too large to be measured by QELS and the nano-alumina size too small to be measured by cryo-SEM (Haridas and Bellare 1995c). The results generated by both the techniques were compared in their overlapping regimes i.e. $400-900 \mathrm{~nm}$ (Haridas and Bellare 1995c), and a close agreement of results to within a $10 \%$ variation within this size range was established. The details on this analysis are reported elsewhere (Haridas and Bellare 1995c).

\section{Results}

\subsection{Viscosity measurements}

Viscosity studies were performed on 1:1 and 1:2 chelate ratio alkoxide samples. The varied parameter was the amount of added water. Water addition was done in increments of $0.2 \mathrm{ml}$ until the occurrence of the sonogelling point. Partially hydrolysed sols were also left undisturbed for up to $210 \mathrm{~min}$ to observe viscosity changes with time, at a fixed water content i.e. keeping the extent of hydrolysis constant. A plot of viscosity versus amount of hydrolysis water added was plotted for each chelate ratio and the results are presented in figures 1 and 2 respectively. Viscosity increases were found to be extremely slow in the initial stages of hydrolysis (i.e. up to $0.2-0.4 \mathrm{ml}$ of water added, depending upon the chelate ratio) but extremely rapid as the water to alkoxide mole ratio approached $3 \cdot 0$. Sonogelling was seen to occur at a water to alkoxide mole ratio of about $3 \cdot 3$ and the viscosity of the chelated systems was about $10,000 \mathrm{cP}$ at the sonogelling point.

\subsection{Optical transmittance measurements}

Optical transmittance measurements were carried out on 1:1 and 1:2 chelate ratio alkoxide samples. The varied parameter was the amount of water added. Water addition was done in increments of $0.2 \mathrm{ml}$ until the occurrence of the sonogelling point 


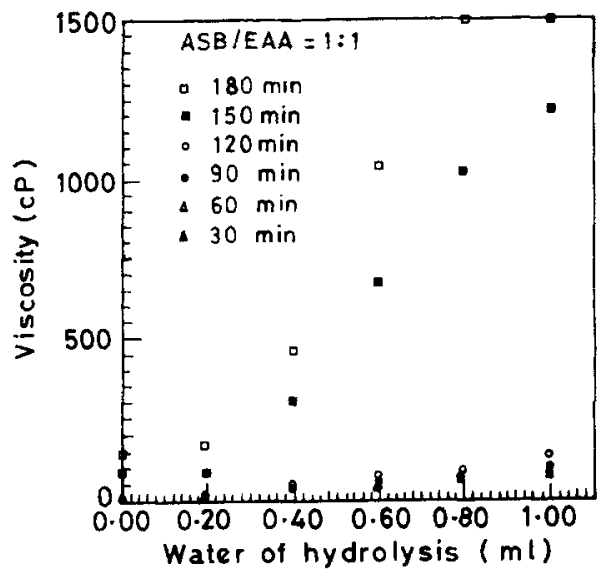

Figure 1. Graph showing the viscosity changes occurring in unchelated and 1:1 chelated, partially hydrolysed alkoxides, with increasing amounts of water. The increase in viscosity is gradual for sols hydrolysed with less than half of the stoichiometric amount of water, and sharp once this value is exceeded.

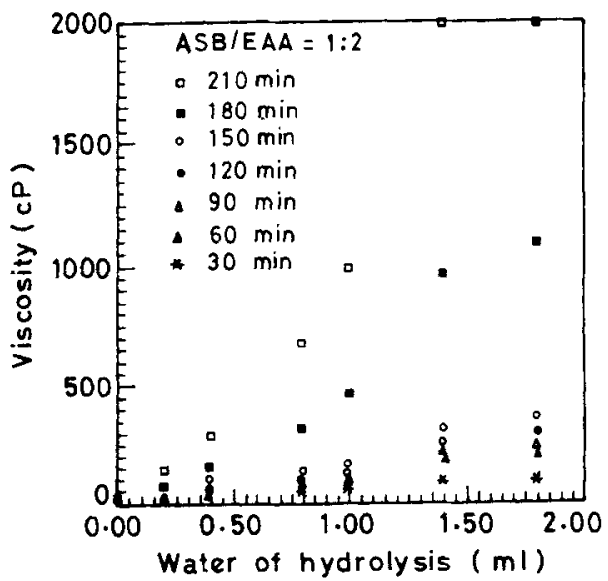

Figure 2. Graph showing the viscosity changes occurring in a $1: 2$ system with increasing amounts of water. The increase in viscosity is gradual for sols with less than $50 \%$ of the hydrolysis water and rapid thereafter.

which occurred at $1.0 \mathrm{ml}$ for a $1: 1$ chelate and $1.8 \mathrm{ml}$ for a $1: 2$ chelate. A plot of optical transmittance, at a representative wavelength of $520 \mathrm{~nm}$ versus amount of hydrolysis water added was plotted for each chelate ratio. The analysis results for both chelate ratios are presented in figure 3 .

\subsection{QELS measurements}

QELS measurements were carried out on unchelated, 1:1 and 1:2 ratio chelated alkoxide samples. The varied parameter was the amount of water added. Water 


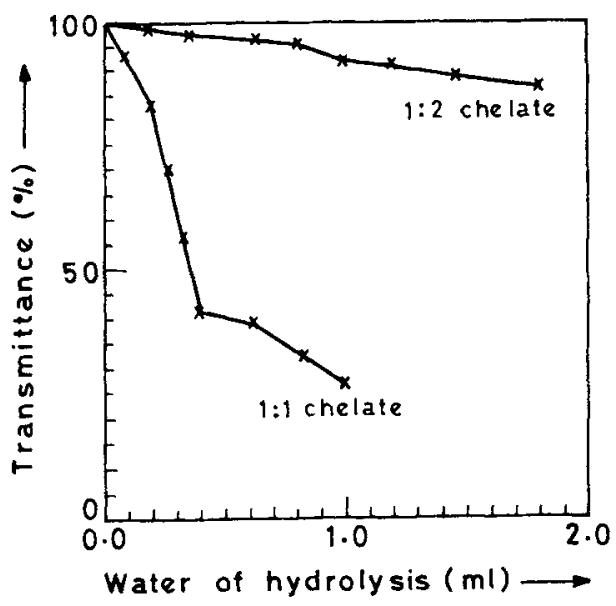

Figure 3. Graph showing the decrease in optical transmittance values with an increase in water content. The decrease is sharp for lower chelate ratios and becomes gradual if this ratio is increased.

Table 1. A comparison of the evolution in alumina size and the accompanied decrease in chelated agglomerate size with varying chelate ratio, for a fixed alcohol content and a constant rate of water addition. Note that the alumina particle size increases per $\mathrm{ml}$ of water added is higher for lower chelate ratios and that higher chelating ratios yield finer particles. Data on the size of the chelated agglomerate in the neighbourhood of the sonogel point could not be measured due to resolution limitations in the cryo-SEM and viscosity limitations in the QELS.

\begin{tabular}{|c|c|c|c|c|c|c|c|}
\hline \multirow[b]{2}{*}{ S. No. } & \multirow[b]{2}{*}{ Water (ml) } & \multicolumn{2}{|c|}{ Unchelated ASB } & \multicolumn{2}{|c|}{$\mathrm{ASB} / \mathrm{EAA}=1: 1$} & \multicolumn{2}{|c|}{$\mathrm{ASB} / \mathrm{EAA}=1: 2$} \\
\hline & & $\begin{array}{c}\text { QELS } \\
\text { (alumina } \\
\text { size) (nm) }\end{array}$ & $\begin{array}{c}\text { SEM } \\
\text { (chelate } \\
\text { size) }(\mathrm{nm})\end{array}$ & $\begin{array}{l}\text { QELS } \\
\text { (alumina } \\
\text { size) (nm) }\end{array}$ & $\begin{array}{c}\text { SEM } \\
\text { (chelate } \\
\text { size) (nm) }\end{array}$ & $\begin{array}{c}\text { QELS } \\
\text { (alumina } \\
\text { size)(nm) }\end{array}$ & $\begin{array}{c}\text { SEM } \\
\text { (chelate } \\
\text { size) (nm) }\end{array}$ \\
\hline 1. & $0 \cdot 0$ & 94 & N. A. & 9 & 6000 & 7 & 8000 \\
\hline 2. & 0.2 & 375 & - & 122 & 3750 & 44 & 4600 \\
\hline 3. & 0.4 & 886 & - & 341 & 2000 & 87 & 3200 \\
\hline 4. & $0 \cdot 6$ & 1450 & - & 672 & 1100 & 116 & 1800 \\
\hline 5. & $0 \cdot 8$ & sonogelled & - & 891 & 900 & 180 & 1400 \\
\hline 6. & $1 \cdot 0$ & - & - & 1046 & 700 & 293 & 900 \\
\hline 7. & $1 \cdot 2$ & - & - & sonogelled & sonogelled & 478 & 750 \\
\hline 8. & 1.4 & - & - & - & - & 642 & 600 \\
\hline 9. & 1.6 & - & - & - & - & 813 & 350 \\
\hline 10. & 1.8 & - & - & - & - & 929 & 182 \\
\hline
\end{tabular}

addition was done in increments of $0.2 \mathrm{ml}$ until the occurrence of the sonogelling point which occurred at $0.6 \mathrm{ml}$ for unchelated samples, $1.0 \mathrm{ml}$ for a $1: 1$ chelated specimen and $1.8 \mathrm{ml}$ for a 1:2 chelated sample. An unchelated sample showed an increase in alumina particle size from $94 \mathrm{~nm}$ at the start of hydrolysis to $1450 \mathrm{~nm}$ at the sonogel point. An increase from $9 \mathrm{~nm}$ to $1046 \mathrm{~nm}$ was seen in 1:1 chelate ratio samples and an increase from $7 \mathrm{~nm}$ to $929 \mathrm{~nm}$ found in 1:2 systems. Particle size measurement results at each water addition are presented in table 1 and a plot of the QELS results versus water 


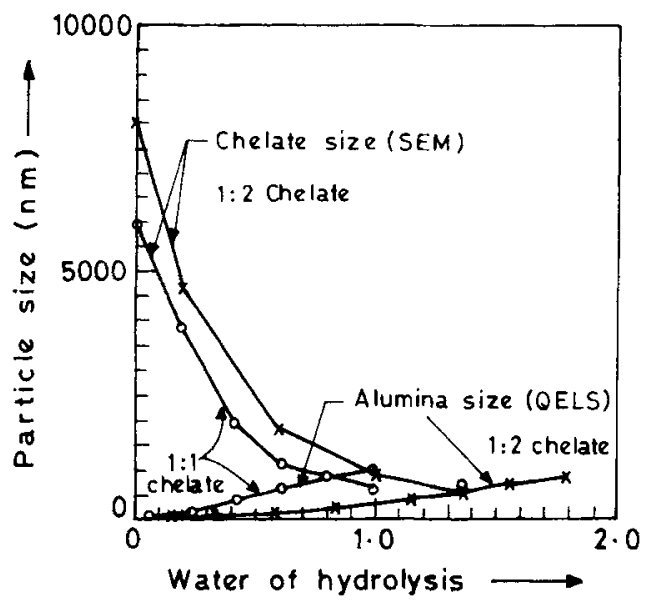

Figure 4. Graph of change in particle size, with water added for a fixed chelate ratio. The schematic shows a decrease in agglomerate size and an increase in nano-alumina size. These were measured by cryo-SEM and QELS respectively. For a fixed volume of hydrolysing water, decreases in agglomerate size are sharper for high ratio chelated samples whereas size increases in nano-alumina are sharper for low ratio chelated samples.

added is shown in figure 4. The QELS variation in figure 4, shows an increase in alumina size as the extent of hydrolysis is increased.

\subsection{Cryo-SEM analyses}

Cryo-SEM analyses were carried out on unchelated, 1:1 and 1:2 ratio chelated alkoxide samples. The varied parameter was the amount of water added. Water addition was done in units of $0.2 \mathrm{ml}$ until the occurrence of the sonogelling point. The cryo-SEM results are presented in figures $5-10$.

To obtain microstructural information at the mixing stage before hydrolysis, the samples were observed by SEM for structural changes caused on chelation. The micrograph in figure 5a is of an unhydrolysed batch without chelating agent. The micrograph is indicative of a true solution being formed on sonicating an alkoxide and shows a 'flat' microstructure, characteristic of a homogeneous, isotropic, liquid phase with no microstructural or morphological detail. Incipient hydrolysis has been eliminated by conducting the process in a glove box under inert gas flow under the influence of a desiccating medium and incorporating alcohol into the sol under continuous ultrasonication conditions. The absence of agglomerated alumina in the micrograph suggests that this control is adequate.

Figure 5 b shows an equimolar ASB/EAA batch hydrolysed by a $60 \mathrm{sec}$ exposure to a $60 \%$ R. H. and corresponds to a chelated alkoxide before hydrolysis. The micrograph shows globular structures in which a high degree of agglomeration is seen. The agglomerated species are thought to be partially hydrolysed alkoxide structures resulting from a controlled hydrolysis. The agglomerated species appear to have a size of about $6-8 \mu$ and have a porous microstructure. The number density of the alumina generated as a result of permitting atmospheric moisture for a controlled time interval (as observed from the QELS patterns) was about 7\%, suggesting that a $60 \mathrm{sec}$ exposure to a $60 \% \mathrm{R}$. H. causes a $7 \%$ hydrolysis to occur. 

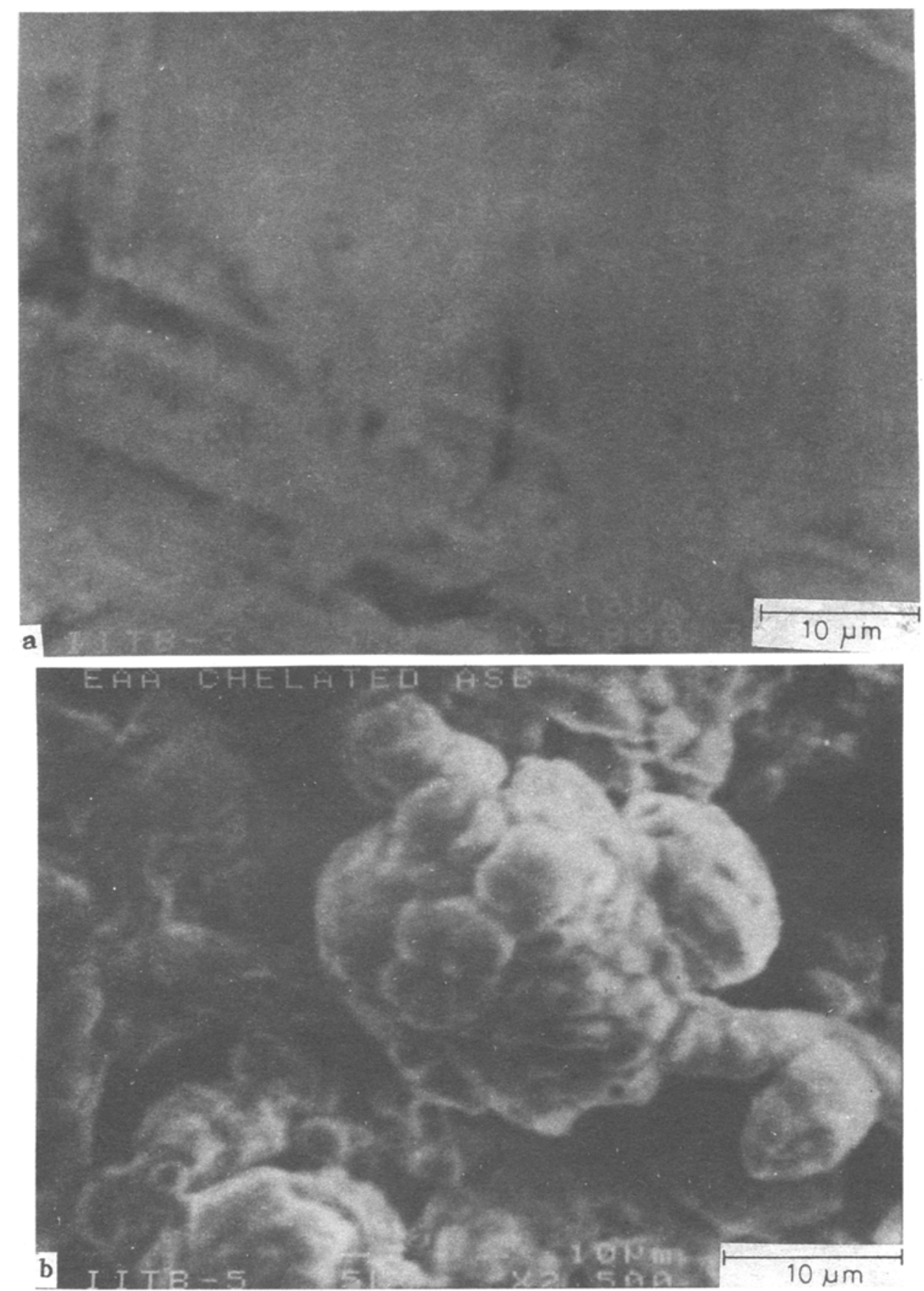

Figure 5. a. Electron micrograph of an unchelated ASB sample showing a flat microstructure characteristic of homogeneous, isotropic liquid phases. The micrograph has been used to confirm that an unchelated alkoxide does not yield microstructural information unless hydrolysed. The absence of imaging artifacts at this step indicates minimal artifactual information being recorded during subsequent processing. The micrograph also suggests a good hydrolysis control over the sol to sonogel conversion indicated by the absence of electron absorbing sites (Bar $=10 \mu \mathrm{m})$ and $\mathbf{b}$. electron micrograph of an alkoxide sample showing the partially hydrolysed agglomerates of ASB caused as a result of a controlled atmospheric hydrolysis in $60 \%$ R.H. conditions in a $0.27 \mathrm{~m}^{3}$ glove box for $60 \mathrm{sec}$. The partially hydrolysed agglomerates are seen to be about $6-8 \mu \mathrm{m}$ in size and are likely to have a porous microstructure $(\mathrm{Bar}=10 \mu \mathrm{m})$. 
An unchelated alkoxide on the addition of $0.4 \mathrm{ml}$, of water, was seen to consist of a bridged network, seen in figure 6a. The micrograph suggests that there is a wide particle size distribution arising from an uncontrolled rate of water attack. The particles show a high degree of agglomeration and the near absence of spherical species is seen in 1:1 chelated systems (figure $6 \mathrm{~b}$ ) with the same amount of water. Under controlled hydrolysis with $0.4 \mathrm{ml}$ of water, the chelated alkoxide globules seen earlier in figure $4 \mathrm{~b}$, were found to break down into finer aggregates under the influence of the ultrasonic probe. Figure $6 \mathrm{~b}$ contains a large number of distribution of spherical species. The chelated agglomerates were seen to shrink in size from $6-8 \mu$ before water addition, to about $3-5 \mu$ at this step. The smaller aggregates had a narrow particle size distribution, as compared to a 1:2 system and the species is seen to be more homogeneous and less porous. A 1:2 system with $0.4 \mathrm{ml}$ as in figure $6 \mathrm{c}$, shows spherical globules measuring about 4 microns in diameter. $0.4 \mathrm{ml}$ of water resulted in uniformly distributed globules with a lower agglomeration number.

After addition of $0.6 \mathrm{ml}$ of water, an unchelated alkoxide system sonogelled. The micrograph of the unchelated sonogel with $0.6 \mathrm{ml}$ of added water in figure $7 \mathrm{a}$, shows pyramidal shaped precipitates of aluminium hydroxide which varied from $1-10 \mu$ indicating a broad particle size distribution. The alumina sonogel was observed to be a highly porous mass. Porosities in the photomicrograph are represented by the dark regions. In comparison however, a 1:1 system on addition of $0.6 \mathrm{ml}$ water showed a preferred orientation of the chelated agglomerates along the arrow shown in figure $7 \mathrm{~b}$ which was noticeably absent in a 1:1 chelated sample with $0.4 \mathrm{ml}$ of added water.

Figure $7 \mathrm{~b}$ shows a lateral thinning i.e. reduction in the dimension perpendicular to the preferred growth direction in agglomerates which appear stretched into a ribbon like form. The chelated species measure about $1 \mu$ in diameter and it is believed that this rapid morphological change is due to the half-sonogelling point being crossed. The agglomerated entities measure about $1-3 \mu$ in diameter. The large globules seen in figure $6 \mathrm{c}$ earlier, appear to have almost broken down with addition $0.6 \mathrm{ml}$ of water.

On adding $1.0 \mathrm{ml}$ of water, the 1:1 chelated sol sonogels. A cryo-SEM of such a sample as shown in figure 8 a reveals the complete dissolution of the chelated agglomerated species seen earlier, and the emergence of a wavy matrix within which, nano-sized aluminium hydroxide particles grow in size to about 1 micron. Thus, gelling of partially hydrolysed chelated precursors is characterized by a thinning mechanism which is not seen in sols chelated prior to a controlled hydrolysis. The 1:2 chelated system however is still in the sol stage at $1.0 \mathrm{ml}$ of added water, whereas the $1: 1$ chelated system has sonogelled. The microstructure of the $1: 2$ chelated system at $1.0 \mathrm{ml}$ of water seen in figure $8 \mathrm{~b}$ reveals an elongated ribbon like structure with a similar lateral thinning in the agglomerated globules. Accompanying this chelate dissolution is the nucleation of nano-alumina already measured to be about $293 \mathrm{~nm}$ by QELS.

When a total of $1.4 \mathrm{ml}$ water was added, the ribbon like microstructure seen in the micrograph of figure $8 \mathrm{~b}$ appears to be further elongated as seen in figure 9 . The tape like structure seen at this stage of hydrolysis was found to evolve from the ribbon-like shape due to a lateral thinning and an increase in the aspect ratio of the chelated agglomerates. As a result of the dissolution, the matrix-chelate phase boundaries do not appear as accentuated as seen in the earlier micrographs. Also seen in inset, is an increase of the alumina particle size, measured by QELS to be $642 \mathrm{~nm}$.

After the addition of $1.8 \mathrm{ml}$ of water to a 1:2 system, the chelated alkoxide sol was observed to sonogel from a free flowing mass, into a rigid monolith, immobilized to an 

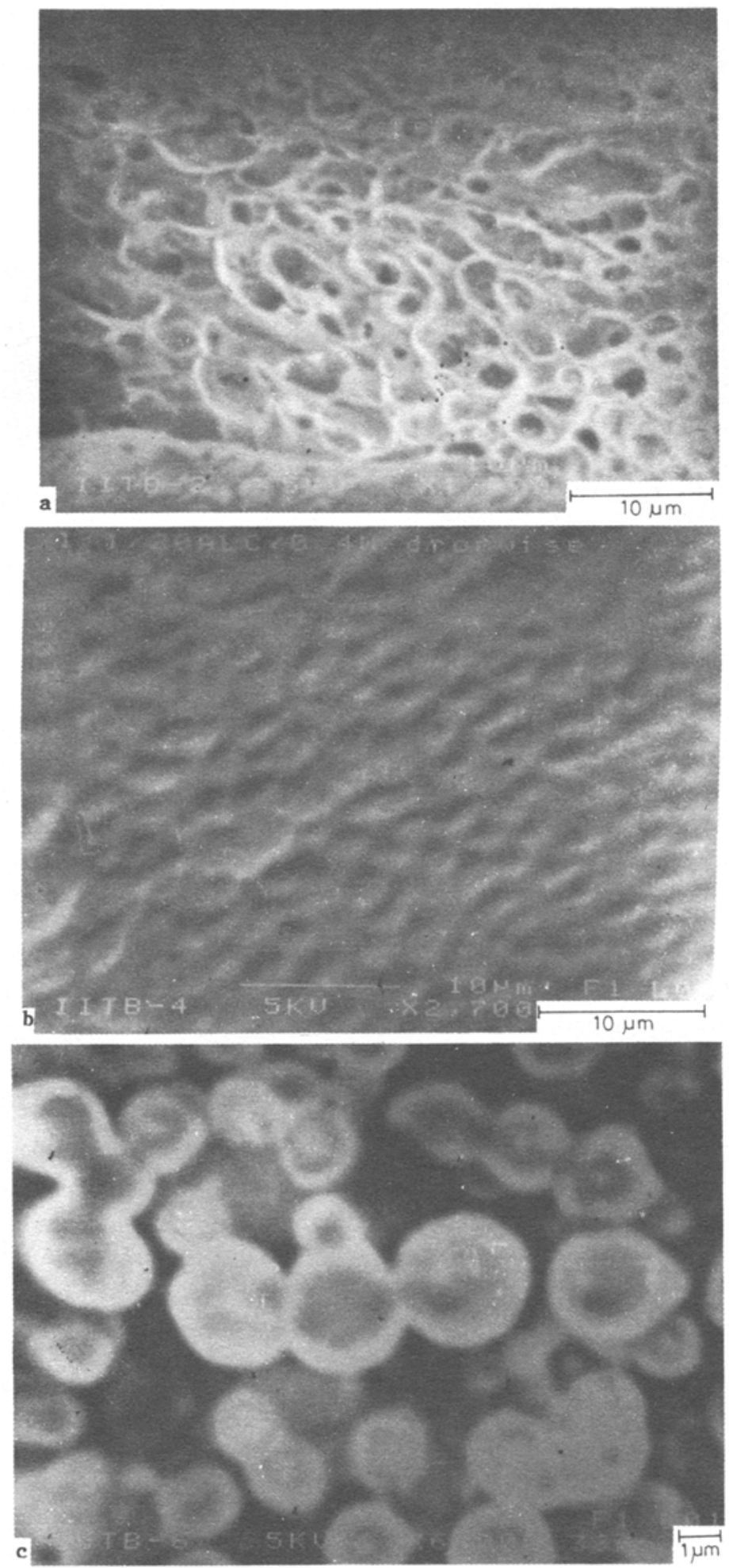
inversion of the sample bottle, with a sharp increase in viscosity to about $10,000 \mathrm{cP}$. The aluminium hydroxide particle size determined by QELS was $929 \mathrm{~nm}$. The electron micrograph of this system as shown in figure 10 was found to consist in a similar wavy matrix pattern as observed at the sonogel point in a 1:1 chelated system, i.e. as shown in figure 8a. Spherical particles of aluminium hydroxide which have evolved from a shrinking, chelated, partially hydrolysed alkoxide are seen in figure 10 . The aluminium hydroxide species lie embedded within the matrix and do not stand out in relief. They are estimated to be about $1 \mu$ in diameter from electron microscopy observations which corroborates the QELS data.

Cryo-SEM particle size results at each water addition are presented in table 1 alongside the QELS data. A plot of cryo-SEM measurements versus water added is shown in figure 4 alongwith the QELS variation. The cryo-SEM variation in figure 4, shows a decrease in chelated agglomerate size with an increase in the extent of hydrolysis.

\section{Discussion}

\subsection{Correlation of measured data}

There is a decrease in percentage transmittance with each water addition for both $1: 1$ and 1:2 chelate ratio samples as seen from figure 3 . However, the decrease in the percentage transmittance in 1:2 samples is less than that in 1:1 systems indicating that with an increase in ethyl acetoacetate content, the extent of hydrolysis drops. QELS data also show that finer particles are generated in a 1:2 chelate than in 1:1 chelate for the same amount of water added.

As the amount of water added to a chelated system is increased, the viscosity of the sol increases first gradually in the initial stages, then sharply as the gelling point is approached. The viscosity increases faster for a 1:1 chelate (figure 1) than for a 1:2 system (figure 2). The gradual viscosity increases in the initial stages, may be due to a break up of the chelated alkoxide on each water addition, without a change in particulate morphology. Towards the final stages, a rapid increase in viscosity arises due to a rapid particle aggregation near the sonogelling point. As seen from the micrographs in figures 7,8 , and 9 , it is evident that after exceeding one half of the sonogelling amount of water, there is a highly preferred orientation of the aggregates. Such systems were seen to gel within $120 \mathrm{~h}$ on atmospheric exposure without any further addition of hydrolysing water. Chelated alkoxides containing less than $50 \%$ of the sonogelling amount of water did not gel even at the end of $1000 \mathrm{~h}$ indicating that $1: 1$ and 1:2 chelates cannot be gelled by exposure to atmosphere and time uniess they contain over 0.5 moles of water per mole of alkoxide. The atmospheric gelation time varied with chelate ratio and the proximity of the hydrolysing system to its sonogelling

Figure 6. a. Micrograph of an unchelated ASB system showing a highly bridged alkoxy network and particles of a wide size distribution formed as a result of uncontrolled hydrolysis $(\mathrm{Bar}=10 \mu \mathrm{m})$, b. micrograph of a 1:1 chelated system showing an agglomerated alkoxide structure measuring about $3-5 \mu \mathrm{m}$ on the addition of $0.4 \mathrm{ml}$ of hydrolysing water. The microstructure appears more uniform and less porous as compared to figure $1 \mathbf{b}(\mathrm{Bar}=10 \mu \mathrm{m})$ and c. a 1:2 chelated system hydrolysed with $0.4 \mathrm{ml}$ of water. Spherical, partially hydrolysed alkoxide agglomerates measuring about $4 \mu \mathrm{m}$ in size are seen $(\mathrm{Bar}=1 \mu \mathrm{m})$. 

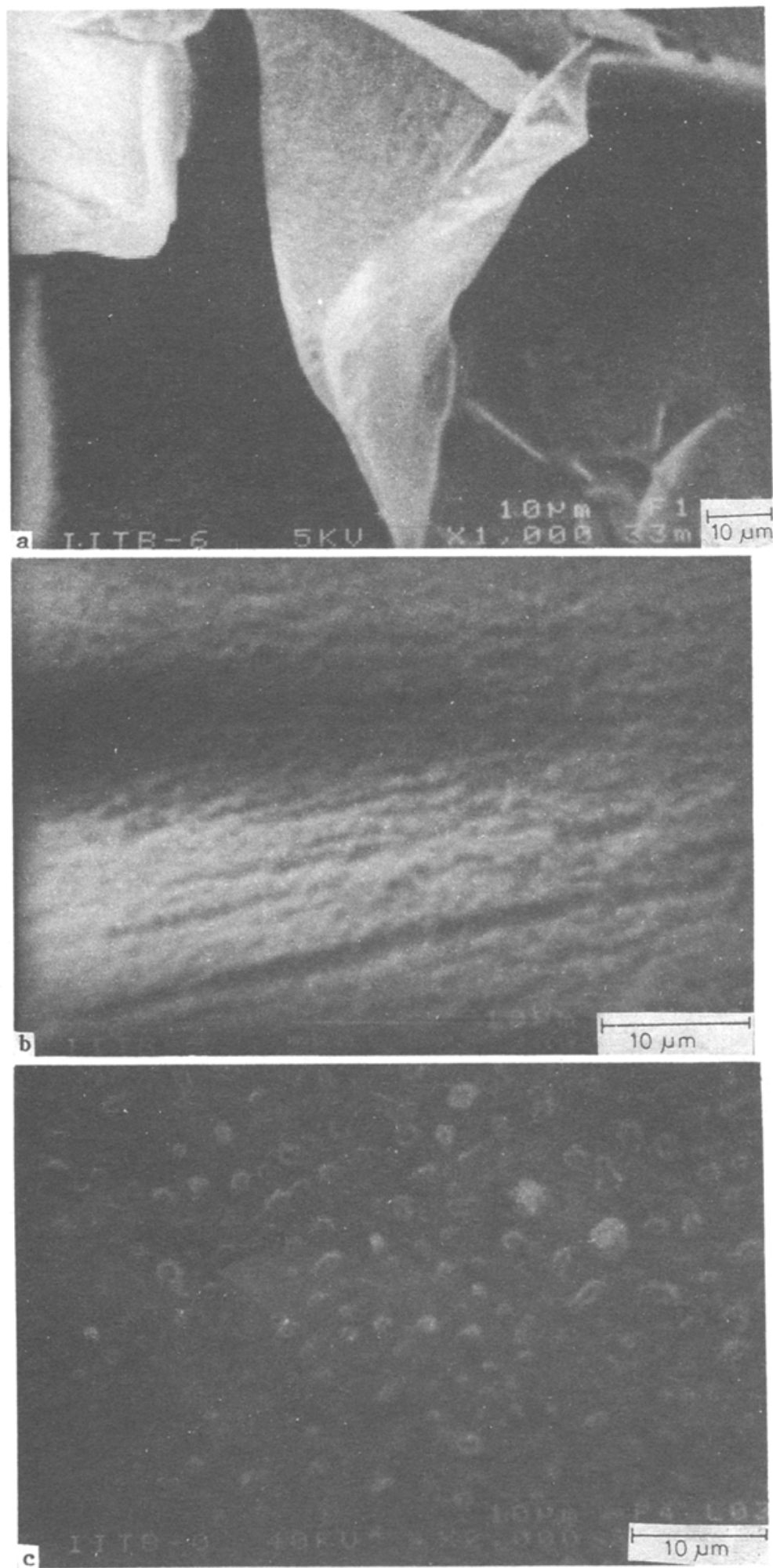

Figure 7. a. Unchelated ASB sonogelling on the addition of $06 \mathrm{ml}$ water. The microstructure is seen as a monolithic pyramid and a highly porous mass with a very broad particle size distribution $(\mathrm{Bar}=10 \mu \mathrm{m})$, b. a 1:1 chelated, partially hydrolysed ASB system approaching the sonogel point on the addition of $06 \mathrm{ml}$ water $(B a r=10 \mu \mathrm{m})$ and $\mathrm{c}$. a $1: 2$ chelated ASB system showing partially hydrolysed agglomerate shrinkage and dissolution at the addition of $0.6 \mathrm{ml}$ water $(\mathrm{Bar}=10 \mu \mathrm{m})$. 

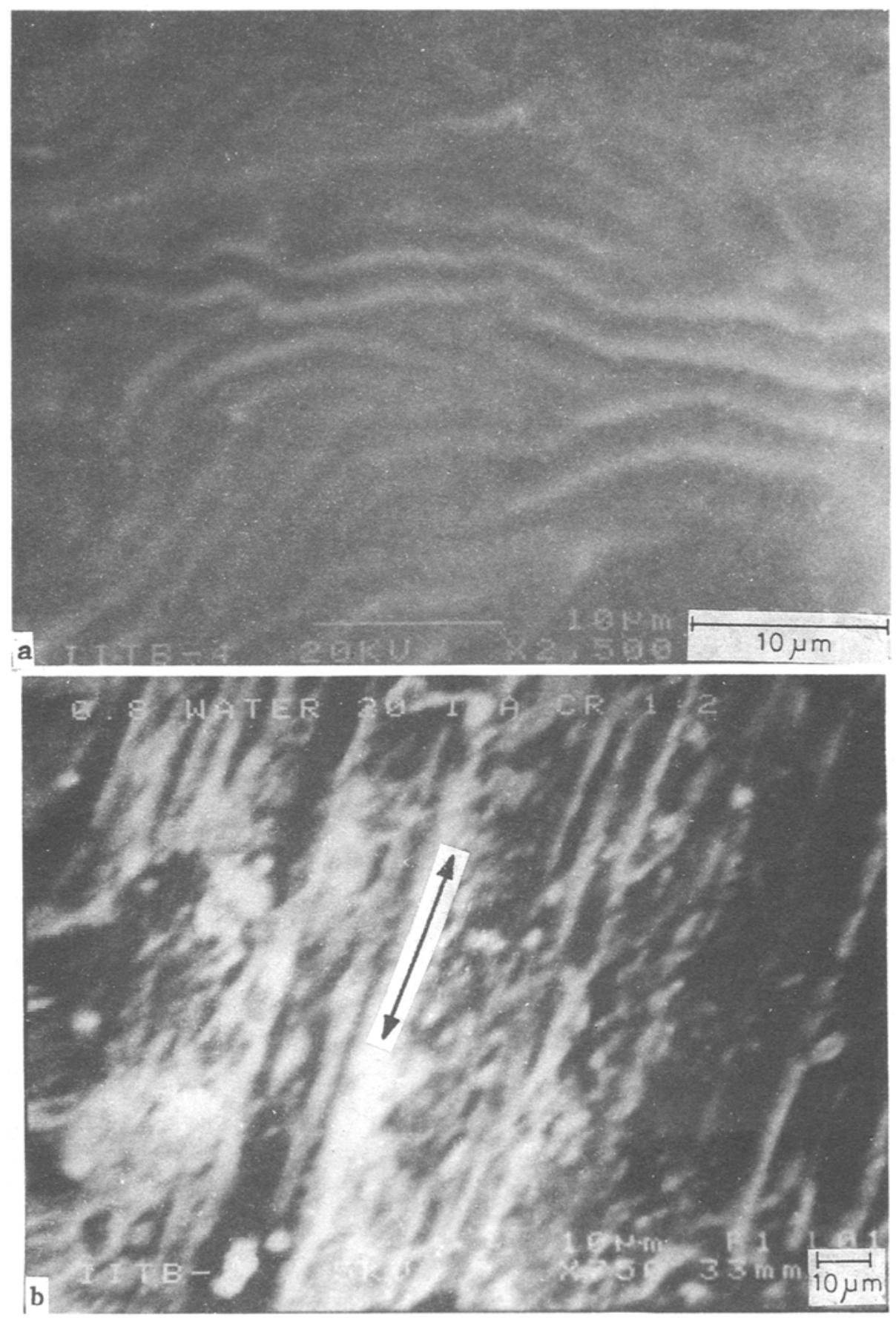

Figure 8. a. A 1:1 chelated, partially hydrolysed system at the point of sonogelling on the addition of $1.0 \mathrm{ml}$ of water $(\mathrm{Bar}=10 \mu \mathrm{m})$, and $\mathbf{b}$. a $1: 2$ chelated, partially hydrolysed system showing an elongation of the agglomerated chelate globules into a ribbon like geometry on the addition of $1.0 \mathrm{ml}$ of water $(\mathrm{Bar}=10 \mu \mathrm{m})$. 


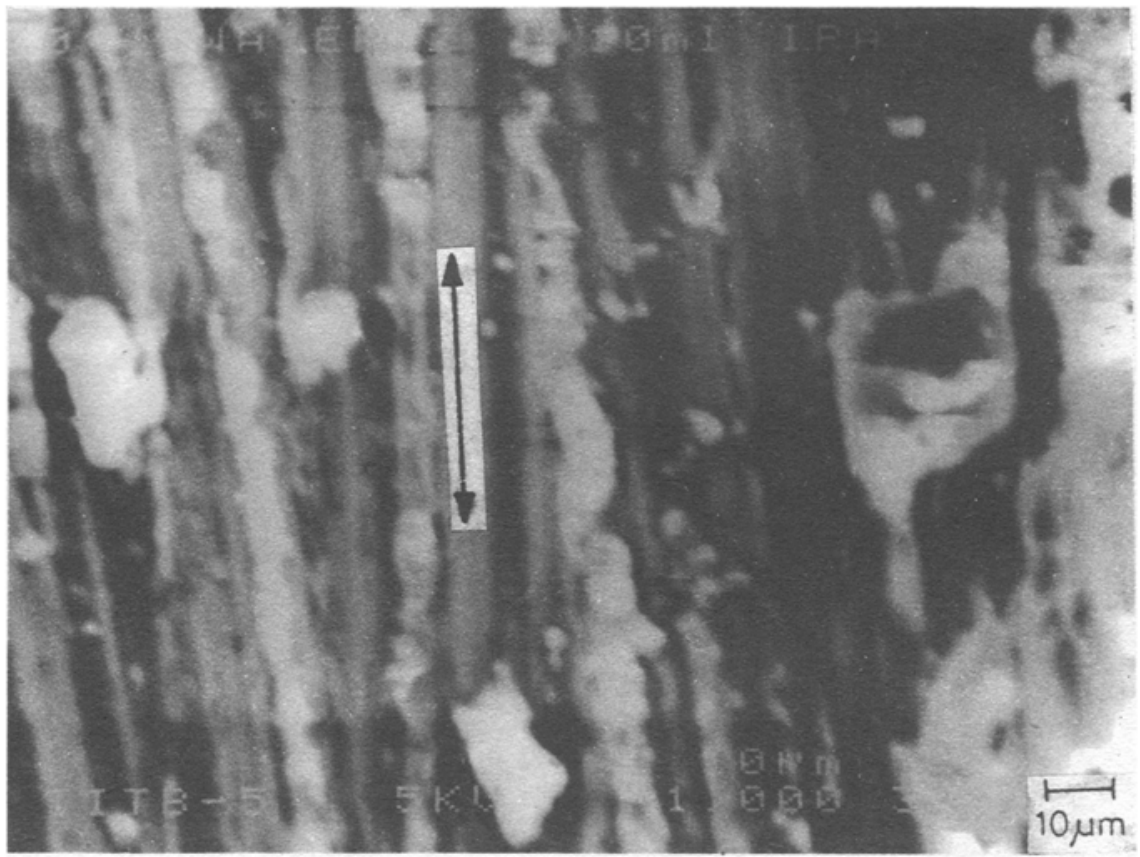

Figure 9. A 1:2 chelated, partially hydrolysed alkoxide possessing a tape like morphology on the controlled addition of $1.4 \mathrm{ml}$ water $(\mathrm{Bar}=10 \mu \mathrm{m})$.

point. The variation in viscosity for 1:1 and 1:2 chelated samples agrees closely with prior work done in this field (Xu et al 1988; Saraswati 1989; Rueb and Zukoski 1992).

\subsection{Sonogelling mechanism observed in chelated alkoxide sols}

The sonogelation mechanism in partially hydrolysed, chelated alkoxide systems is different from that in peptised alumina sols, where edge to face interaction takes place between plate like particles. In case of a chelated system the alumina particles generated are thought to be separated from each other by a steric barrier instead of a surface charge. Ethyl acetoacetate is known to act as a surfactant and its ability to inhibit agglomeration documented (Nass and Schmidt 1989, 1990). Thus, the alumina formed, is stabilized by ethyl acetoacetate and excessive or rapid aggregation and precipitation prevented. On evaporation of the solvent, a transparent, clear sonogel is formed. From rheological considerations, the increase in the viscosity is found to be in good agreement with the experimental values reported in literature (Xu et al 1988; Rueb and Zukoski 1992) and that, there is a very small difference in viscosities in unchelated systems and chelated systems at their sonogelling point.

Based on data measured by the characterization techniques, a primitive model showing the morphological changes occurring during the sonogelling of a chelated alkoxide sol has been developed and is shown in figure 11. As can be seen from the schematic model, before the addition of any hydrolysing water, only chelated,

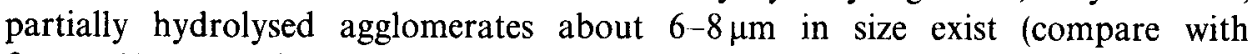
figure $5 \mathrm{~b}$ ). By adding 0.4 ml water to a 1:2 system, the agglomerate size is reduced 


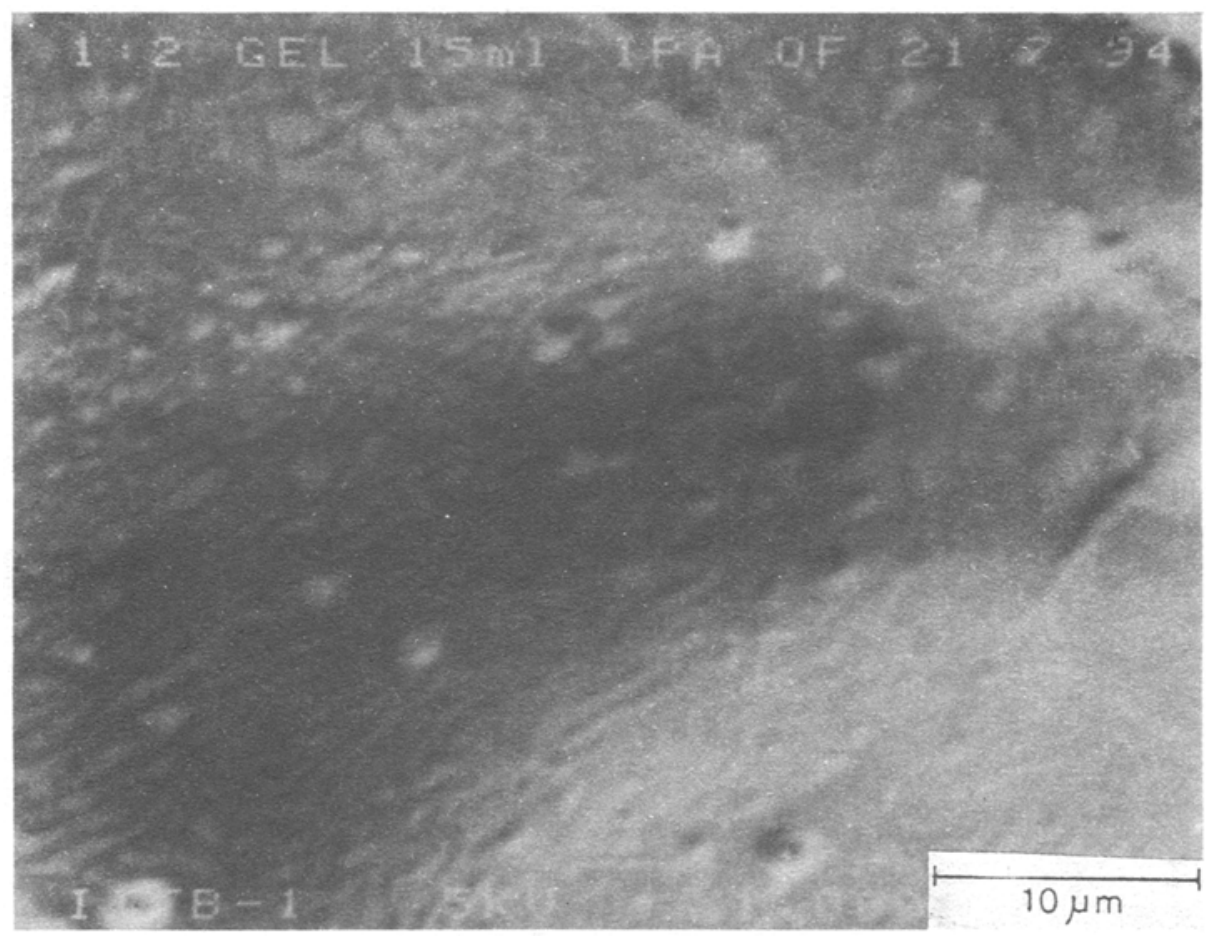

Figure 10. A 1:2 chelated, partially hydrolysed alkoxide system sonogelling on the addition of $1.8 \mathrm{ml}$ of water $(\mathrm{Bar}=10 \mu \mathrm{m})$.

and nano-alumina production starts as noticed in figure $6 \mathrm{c}$. When $0.6 \mathrm{ml}$ water is added to this system, the agglomerates assume a cylindrical shape, as seen in figure $7 \mathrm{c}$, whereas the alumina particles increase in size. At $1.0 \mathrm{ml}$ of water addition, the agglomerates take a ribbon like form and reduce in diameter as seen in figure $8 \mathrm{~b}$. Alumina grows in size. At $1.2 \mathrm{ml}$ of water, the ribbons elongate into slender tapes and the alumina acquires sub-micron dimensions. At 1.4 $\mathrm{ml}$ of water, the tapes undergo lateral thinning (compare with figure 9) while alumina is steadily increasing in size as a result of accelerated dechelation. At $1.6 \mathrm{ml}$ of water, the tapes have almost dissolved into the matrix and alumina has acquired micron dimensions. At $1.8 \mathrm{ml}$ of water, the $1: 2$ chelated, partially hydrolysed system converts to a sonogel. The agglomerated species have dissolved into the matrix and alumina has grown to its final size, as seen in figure 10. There is a rapid increase in viscosity until the sol turns to a rigid monolith. From the model suggested in figure 11, and other supporting data described in this paper, it may be summed up that the microstructure evolution in a chelated alkoxide system adopts the following metamorphic path on controlled hydrolysis: partially hydrolysed, chelated species agglomerate into near spherical geometries, agglomerated species elongate into a cylindrical form, the cylinders stretch into ribbon like structures on a lateral thinning of the agglomerated assemblies, the chelated entities dissolve, nano-aluminium hydroxide nucleates and aluminium hydroxide particles grow into near spherical shapes on increased water addition. The partially hydrolysed chelated alkoxide, which acts as a secondary electron absorbing site, is found to reduce in size and change in shape. In addition to the unreacted alkoxide component, the partially hydrolysed species is 

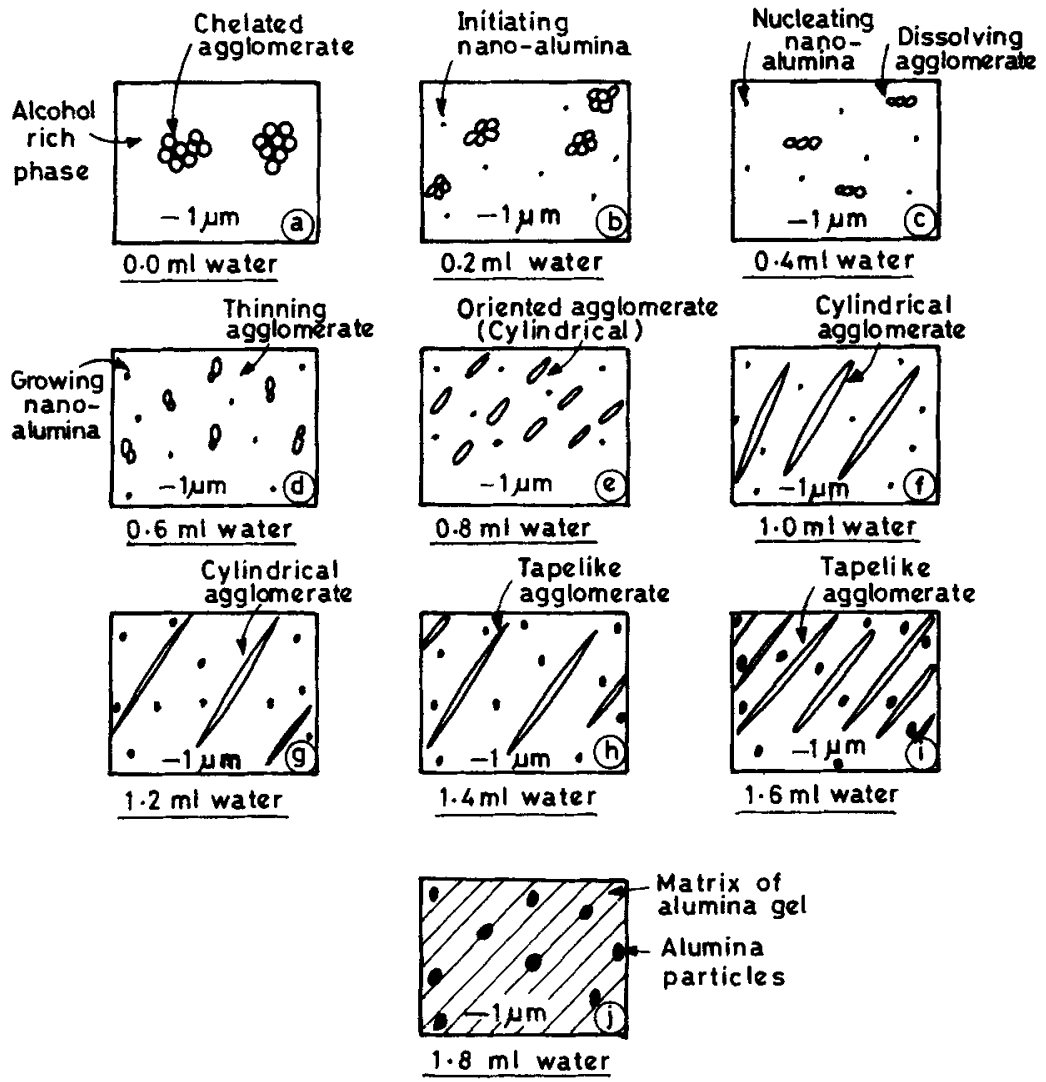

Figure 11. A schematic gelling model depicting the morphological changes occurring in a chelated, partially hydrolysed alkoxide system on the controlled addition of water. a. Before the addition of water, only chelated, partially hydrolysed agglomerates about 6-8 $\mu$ in size exist, b. at $0.4 \mathrm{ml}$ water addition, the agglomerates reduce in size and nano-alumina production starts, c. at $0.6 \mathrm{ml}$ water addition, the agglomerates assume a cylindrical shape whereas the alumina particles increase first in number and then in size, $\mathbf{d}$. at $1.0 \mathrm{ml}$ water addition, the agglomerates take a ribbon like form and reduce in diameter. Alumina grows in size, e. at $1.2 \mathrm{ml}$ water, the ribbons elongate into slender tapes and the alumina acquires sub-micron dimensions, f. at $1.4 \mathrm{ml}$ water, the tapes undergo lateral thinning to nanometer sized diameters while alumina is steadily increasing in size as a result of accelerated dechelation, g. at $1.6 \mathrm{ml}$ water, the tapes have almost dissolved into the matrix and alumina has acquired micron dimensions, and $\mathbf{h}$. at $1.8 \mathrm{ml}$ water, the $1: 2$ chelated, partially hydrolysed system sonogels. The agglomerated species have dissolved into the alcoholic matrix and alumina has grown to its final size. There is a rapid increase in viscosity until the sol turns to a rigid monolith.

a source of alumina which is seen to grow in size and distribution on the dissolution of the controllably hydrolysed species.

4.3 An evaluation of the suggested sonogelling mechanism versus currently known sonogelling mechanisms

The sonogelling mechanism proposed in this paper is substantially different from the Zarzycki model (Zarzycki 1987, 1990, 1991) which, in the authors opinion, is valid only 
for unchelated species. Zarzycki's growth mechanisms predict the development of a 'wispy' microstructure with an increase in the extent of hydrolysis and the proximity of the system to its gelling point.

The Zarzycki mechanism suggests that structure of the gel depends upon the nature of the catalyst used, if any: acid catalysis leads to filamentous structures with a low degree of reticulation of the $-\mathbf{M}-\mathrm{O}-\mathrm{M}$ - chains, while base catalysis produces more compact spheroidal particles with a high internal degree of reticulation. The interstitial liquid, as per the Zarzycki model contains unreacted sol particles which progressively attach themselves to the network. Furthermore, there is a transport of alumina particles from convex to concave parts due to solubility effects based on Thomson's relation (Zarzycki 1987) which smoothes the inequalities between linked particles and converts the chains into filaments. Additional deposition of aluminium hydroxide from solution may further 'nourish' and thus stiffen the chains. The syneresis effect then sets in whereby the network slowly contracts and tends to progressively expel the interstitial liquid in order to reduce the internal interface. As a result, the gel microstructure is found to grow increasingly viscous, cross-linked and 'wispy'.

From the evidences presented in this paper, it is felt that Zarzycki's wispy (or networked) microstructures (Zarzycki 1991) represent a small part of the structural evolution in chelated alkoxides i.e. they represent the evolution only in aluminium hydroxide and ignore the microstructure development in chelated agglomerate species. In the proposed sonogelling mechanism, the authors demonstrate that chelated agglomerates dissolve into the alcoholic matrix through a range of microstructures while aluminium hydroxide species nucleate. Consequently, the microstructure becomes 'decreasingly lumpy and increasingly wispy' as agglomerate size decreases and alumina size increases with every increase in the extent of hydrolysis.

\section{Conclusions}

A schematic model indicative of the mechanism of sonogel formation in chelated alkoxide sols has been proposed. The chelation mechanism follows a dissolutionnucleation kinetics with the morphology and size of both the chelated species as well as the aluminium hydroxide being formed, changing at every water addition during hydrolysis. Electron microscopy has revealed that the spherical chelated entities elongate into a cylindrical shape with a preferred growth direction. When the hydrolysing water has exceeded half the sonogelling amount of water, the cylindrical morphology is seen to flatten into a ribbon-shaped structure with nano-aluminium hydroxide developing at each water assisted dechelation. This results in a size reduction of the chelated agglomerates. At the sonogel point, the aluminium hydroxide particles have a size which is chelate ratio dependent. A higher chelate ratio is seen to yield finer particles.

The rate of hydrolysis in a 1:1 chelate is higher than that in a 1:2 chelate and hence results in coarser particles. The increase in viscosity for a 1:1 system is more rapid as compared to a 1:2 system and consequently, the microstructure evolves more slowly in higher chelate ratios. The globular chelated species break down into finer aggregates and simultaneously, transparent nano-alumina aggregates into larger entities. Both processes are simultaneous and mutually related and serve as useful indicators in evaluating the microstructure development in chelated alkoxide precursors. 
Alumina sonogels having a stable transmittance of over $90 \%$ have, for the first time, been made from a high EAA/ASB chelate ratio, high alcohol, slow water addition and vigorous sonication. The sol morphology of the water unchelating, chelated alkoxide changes at every water addition, as does the viscosity. The viscosity changes in chelated alkoxide systems have been found to be consistent with previously reported values for unchelated alkoxides (Xu et al 1988; Rueb and Zukoski 1992).

\section{References}

Babonneau F, Coury L and Livage J 1990 J. Non-Cryst. Solids 121153

Bellare J R 1988 Cryo-electron and optical microscopy of surfactant microstructures, Doctoral Dissertation, University of Minnesota, USA

Bhadriraju K and Bellare J R 1993 Proc. Microsc. Soc. Am. 51235

Ching J Y C and Klein L C 1988a J. Am. Ceram. Soc. 7183

Ching J Y C and Klein L C 1988b J. Am. Ceram. Soc. 7186

Debsikdar J C 1985 J. Mater. Sci. 204454

Finsy R 1994 Adv. Colloid \& Interface Sci. 5279

Haridas M M and Bellare J R 1995a A process to synthesize highly transparent sonogels from chelated alkoxides, Indian patent application No. 487/BOM/95

Haridas M M and Bellare J R 1995b A method to produce nordstrandite from chelated aluminium alkoxides, Indian patent application No. 488/BOM/95

Haridas M M and Bellare J R 1995c Proc. 3rd international engineering foundation conference (eds.)Brij Moudgil and Pradip (Pune: Tata Research Development and Design Centre) pp 17-21 (to be published)

Haridas M M and Bellare J R 1996 Ceram. Int. (accepted)

Haridas M M, Menon A, Goyal N, Chandran S and Bellare J R 1995 Ceram. Int. 22155

Heinrich T, Raether F and Marsmann H 1994 J. Non-Cryst. Solids 16814

Ichinose N, Ozaki Y and Kashu S 1992 in Superfine particle technology (London: Springer-Verlag) 1st ed. Jahn W and Strey R 1988 J. Phys. Chem. 922294

Jones R W 1989 in The fundamental principles of sol-gel technology (London: Inst. of Metals) 1st ed.

Leaustic A and Riman R E 1991 J. Non-Cryst. Solids 135259

Lee J W, Won C W and Chun B S 1990 J. Korean Inst. Met. 28437

Mehrotra R K and Mehrotra R C 1961 Canadian J. Chem. 39795

Nass R and Schmidt H J 1989 Proc. 2nd. int. conf. on ceramic powder processing science (ed.) H Hausner (Cologne: Publ. Deutsche Keramische Gesellschaft) pp 69-73

Nass R and Schmidt H J 1990 J. Non-Cryst. Solids 121329

Ogihara T, Nakajima H, Yanagawa T, Ogata N and Yoshida K 1991 J. Am. Ceram. Soc. 742263

Rueb C J and Zukoski C F 1992 Mater. Res. Soc. Symp. Proc. 249279

Sakka S and Yoko T 1991 Ceram. Int. 17217

Sanchez C, Livage L, Henry M and Babonneau F 1988 J. Non-Cryst. Solids 10065

Saraswati V 1989 Key Eng. Mater. 29593

Schmidt H 1988 J. Non-Cryst. Solids 10051

Segal D 1989 in Chemical synthesis of advanced materials (Cambridge: Cambridge University Press) 1st ed. Strawbridge I and James P F 1986 Br. Ceram. Proc. 38251

Wengrovius J H, Garbauskas M F, Williams E A, Going R C, Donahue P E and Smith J F 1986 J. Am. Chem. Soc. 108982

Xu R, Pope E J A and Mackenzie J D 1988 J. Non-Cryst. Solids 106242

Yogo T and Iwahara H 1992 J. Mater. Sci. 271499

Yoldas B E $1973 \mathrm{~J}$. Appl. Chem. Biotechnol. 23803

Yoldas B E 1975 Ceram. Bull. 54 286, 289

Zarzycki J 1987 J. Non-Cryst. Solids $95-96173$

Zarzycki J 1990 J. Non-Cryst. Solids 121110

Zarzycki J 1991 in Glasses and the vitreous state (Cambridge: Cambridge University Press) 